\title{
THE LAMB SHIFT AND ULTRA HIGH ENERGY COSMIC RAYS
}

\author{
SHE-SHENG XUE \\ I.C.R.A. and Physics Department, University of Rome "La Sapienza", I-00185 \\ Rome, Italy \\ E-mail: xue@icra.it
}

\begin{abstract}
On the analogy with the Lamb shift, we study the vacuum effect that proton's electric field interacts with virtual particles in the vacuum. We find a possible quantum instability that triggered by an external force, proton's electric field interacting with virtual particles spontaneously induces a quantum force that back reacts on the proton in the direction of the external trigger force. Such a quantum-induced force accelerates the proton runaway, by gaining the zero-point energy from the vacuum $\left(\sim 10^{-5} \mathrm{eV} / \mathrm{cm}\right)$. This effect possibly accounts for the mysterious origin and spectrum of ultra high-energy cosmic ray (UHECR) events above $10^{20} \mathrm{eV}$, and explains the puzzle why the GZK cutoff is absent. The candidates of these events could be primary protons from the early Universe.
\end{abstract}

The effective Lagrangian for a proton. The Lamb shift ${ }^{1}$ shows that the energy level $2 S_{\frac{1}{2}}$ of the hydrogen atom spectrum is shifted upward $+1008 \mathrm{MHz}$, compared with $2 P_{\frac{1}{2}}$. This implies that QED vacuum effects drain the zeropoint energy to a hydrogen atom. We relate these vacuum effects to the origin of UHECR events.

Considering a proton interacting with virtual particles in the vacuum, we introduce (i) $\Psi$ and $A_{\mu}$ describing a proton field and its gauge potential; (ii) $\psi_{q}$ and $A_{\mu}^{q}$ describing the quantum fields of virtual fermions and photons in the vacuum. To study this system, we start with a renormalized lagrangian density $L(x)$ with all necessary renormalization counterterms,

$$
\begin{aligned}
L(x) & =-\frac{1}{4}\left(F^{2}+F_{q}^{2}\right)+\bar{\Psi}\left[i \gamma^{\mu} \partial_{\mu}-m_{p}-e_{p} \gamma^{\mu}\left(A_{\mu}+A_{\mu}^{q}\right)\right] \Psi \\
& +\bar{\psi}_{q}\left[i \gamma^{\mu} \partial_{\mu}-m-e \gamma^{\mu}\left(A_{\mu}+A_{\mu}^{q}\right)\right] \psi_{q}+(\text { c.t. }),
\end{aligned}
$$

where $F$ and $F_{q}$ are classical and quantum electromagnetic field tensors, $e$ and $m\left(e_{p}\right.$ and $\left.m_{p}\right)$ and are electron(proton) charge and mass. This is a complex interacting system, the classical fields $\Psi$ and $A_{\mu}$, quantum fields 
$\psi_{q}$ and $A_{\mu}^{q}$ are coupled together. To the first order, we obtain an effective interacting lagragian (details will be presented elsewhere):

$$
\begin{aligned}
L_{i}^{\mathrm{ext}}(x) & =-\bar{e} A_{\nu}^{\mathrm{ext}} \bar{\Psi} \gamma^{\nu} \Psi, \quad \bar{e}=e_{p} \frac{4 \alpha^{2}}{3}\left(\ln \frac{m}{\mu}-\frac{7}{40}\right) ; \\
A_{\nu}^{\mathrm{ext}}(x) & =\int \frac{d^{4} x^{\prime}}{m^{2}} \operatorname{tr}\left[S_{F}\left(x-x^{\prime}\right) \gamma_{\mu} S_{F}\left(x^{\prime}-x\right) \gamma_{\nu}\right] A^{\mu}\left(x^{\prime}\right), \\
& =\frac{1}{60 \pi m^{4}}\left(g_{\mu \nu}-\frac{\partial_{\mu} \partial_{\nu}}{\Delta}\right)(\Delta)^{2} A^{\mu}(x) .
\end{aligned}
$$

In Feynman's prescription of particles and antiparticles, Eq.(3) shows that a pair of virtual fermion and antifermion (virtual pair) is created at one spacetime point $x$ and annihilates at another $x^{\prime}$, behaving as an electric dipole $\vec{P}$ in its life-time. This virtual pair couples to the classical field $A^{\mu}\left(x^{\prime}\right)$ of the proton at $x$. As a result, an induced quantum dipole field $A_{\nu}^{\text {ext }}(x)$ is created, attributed to virtual pairs, and this quantum field back interacts with the proton as an external field $A_{\mu}^{\text {ext }}\left(\vec{E}^{\text {ext }}\right)$.

Induced quantum force and instability. In the case of the proton at rest or traveling with a constant velocity, we might conclude $A_{\mu}^{\mathrm{ext}}(x) \equiv 0$ for its transversality and the Lorentz invariance: $A^{\mu}(x)=\frac{e_{p}}{4 \pi|\vec{x}|} g^{\mu \circ}$, being longitudinal in an instantaneous rest frame of the proton. However, we have to consider the quantum nature of quantum-induced field $A_{\mu}^{\text {ext }}(x)$. In the absence of an external field, the quantum-field fluctuations of virtual pairs and their dipole fields are entirely random-fluctuating of a time-scale $\delta \tau_{q} \sim 1 / m$ in the spacetime, we do not expect any quantum-induced field of life-time $>\delta \tau_{q}$ in(at) any particular direction(point) of the spacetime. In the presence of longitudinal electric field of the proton, (i) the transverse quantum-field fluctuations of virtual pairs and their dipole fields $A_{\mu}^{\text {ext }}(x)$ are entirely random-fluctuating of a time-scale $\delta \tau_{q} \sim 1 / m$ in the spacetime; (ii) the longitudinal quantum-field fluctuations of virtual pairs and their dipole fields $A_{\mu}^{\text {ext }}(x)$, although their life-time can be larger than $\delta \tau_{q}$, are entirely spherically symmetric and total dipole field $A_{\mu}^{\mathrm{ext}}(x)$ acting on the proton is zero. Thus, indeed we do not expect any induced quantum field $A_{\mu}^{\text {ext }}(x)$ of life-time $>\delta \tau_{q}$, acting on the proton in a peculiar direction of the instantaneous rest frame.

Nevertheless, in the instantaneous rest frame of the proton, we consider the case that an external trigger force $\vec{F}_{t r i}$ accelerates the proton for a time interval $\Delta t_{t r i} \gg \delta \tau_{q}$. As a result, proton's electric field $\vec{E}\left(x^{\prime}\right)$ gets a transverse component $\vec{E}_{\perp}\left(x^{\prime}\right)$, whose distribution (both value and direction) is axial symmetric with respect to the direction of $\vec{F}_{t r i}$, as given by the Lien- 
ard Wiechert field. This transverse field induces the transverse component $\vec{P}_{\perp}\left(x^{\prime}\right)$ of quantum electric dipoles of virtual pairs at $x^{\prime}, \vec{P}_{\perp}\left(x^{\prime}\right) \sim \vec{E}_{\perp}\left(x^{\prime}\right)$. The spatial distribution of $\vec{P}_{\perp}\left(x^{\prime}\right)$ is the same as that of $\vec{E}_{\perp}\left(x^{\prime}\right)$. These quantum electric dipoles create electric dipole fields $\vec{E}_{\perp}^{\text {ext }}(x)$ (4) back reacting on the proton at $x$. Summing over all contributions of quantum electric dipoles $\vec{P}_{\perp}\left(x^{\prime}\right)$ of virtual pairs, we find that the total $\vec{E}_{\perp}^{\text {ext }}(x)$ acts on the proton in the same direction of $\vec{F}_{t r i}$. This total $\vec{E}_{\perp}^{\text {ext }}(x)$ acting on the proton then plays the same role of $\vec{F}_{t r i}$. This implies that quantum-field fluctuations of virtual pairs, triggered by $\vec{F}_{t r i}$, could cause a quantum runaway instability that the proton is accelerated further and further by a quantum force $\vec{F}_{q}=\vec{e} \vec{E}_{\perp}^{\text {ext }}(x)$ even after the trigger force is off. Such a quantum instability can take place, provided quantum electric dipoles $\vec{P}_{\perp}\left(x^{\prime}\right)$ and their electric fields $\vec{E}_{\perp}^{\text {ext }}(x)$ have a life-time $\gg \delta \tau_{q}$. For the Lorentz invariance and homogeneity of the vacuum state, the quantum-induced field $\vec{E}_{\perp}^{\text {ext }}$ or $F_{\mu \nu}^{\text {ext }}$ must be constant.

In the following, we adopt a semi-classical model to qualitatively estimate the value of such an induced quantum driving force in the instantaneous rest frame of the proton. Virtual pairs in an external field can be possibly considered as unstable excitations of bound states of virtual fermions and antifermions. We approximately estimate their binding energy, size and life-time. The energy scale of quantum-field fluctuations of virtual pairs must be much smaller than the electron mass $m$, otherwise real electrons and positrons would be created. We thus adopt a non-relativistic description for virtual pairs, whose size is about $\frac{2}{\alpha m}$, binding energy $\sim \frac{\alpha^{2} m}{2}$. This indicates the size of electric dipoles $|d| \sim\left|x-x^{\prime}\right| \sim \frac{2}{\alpha m}$, and $\vec{P}=|e| \vec{d}$ in Eq.(3). The cross-section(probability) of the annihilation and creation of such a virtual pair is about $\pi\left(\frac{\alpha}{m}\right)^{2}$. The life-time of such a virtual pair is then $\delta \tau_{p} \sim \frac{2}{\alpha^{5} m}=6.2 \cdot 10^{-11} \mathrm{sec}$. This indicates the life-time of quantum electric dipole $\vec{P}_{\perp}$ and field $\vec{E}_{\perp}^{\text {ext }}(x)$ is $\delta \tau_{p} \sim \frac{2}{\alpha^{5} m}$, which is much larger than $\delta \tau_{q} \sim \frac{1}{m}$. Using $\vec{P}_{\perp} \cdot \vec{E}_{\perp} \lesssim e^{2} /(4 \pi|d|)$, we can estimate $\left|\vec{E}_{\perp}\right| \lesssim|e| /\left(4 \pi|d|^{2}\right)$.

The large wavelength modes $k$ of proton's gauge field $A_{\mu}$ are sensitive to the low-lying states of virtual pairs of size $\sim \frac{2}{\alpha m}$. This suggests $k \sim \frac{\alpha m}{2}$ in Eq.(4) and the infrared cutoff $\mu \sim \frac{\alpha m}{2}$ in Eq.(2). The amplitudes of the induced quantum dipole fields $A_{\mu}^{\text {ext }}$ and $\vec{E}^{\text {ext }}$ are approximately given by,

$$
A_{\mu}^{\mathrm{ext}} \simeq \frac{\alpha^{4}}{960 \pi} A_{\mu} ; \quad \vec{E}_{\perp}^{\mathrm{ext}} \simeq \frac{\alpha^{4}}{960 \pi} \vec{E}_{\perp}
$$

Summing over the angular distribution of virtual pairs, we obtain the spon- 
taneously induced quantum force:

$$
\vec{F}_{q}=\frac{\delta E}{\delta \vec{x}}=\bar{e} \vec{E}_{\perp}^{\mathrm{ext}} \simeq 2.82 \cdot 10^{-5}(\mathrm{eV} / \mathrm{cm}) \vec{u},
$$

and its direction $\vec{u}(|\vec{u}|=1)$ is kept in the same direction of proton's acceleration. The life-time of this induced quantum force is $\delta \tau_{p} \sim \frac{2}{\alpha^{5} m} \gg \tau_{q}$, and it seems that the quantum instability ought to occurs. Eq.(6) holds for any charged particles and can be experimentally tested in a laboratory. The estimations and considerations are still very qualitative and speculative, need to be further improved and verified. It is highly deserved to have a quantitative computation of this quantum-induced force and instability.

Vacuum energy gain and lost. We turn to discuss Eq.(6) from the energetical point of view. In the absence of any external field, the quantum-field fluctuations of virtual pairs are entirely random in the spacetime. This determines the maximum value of the zero-point energy. However, in the presence of a proton and its external field that couples to virtual pairs, the quantum-field fluctuations of virtual pairs are re-oriented towards the direction of the external field, so that the zero-point energy is reduced. The variation of the zero-point energies due to the longitudinal component of the external field dissipates back to the vacuum and the external field. While, the variation of the zero-point energies due to the transverse component of the external field drains to the proton as a recoiling effect. This recoiling effect is realized by an induced quantum field $A_{\mu}^{\text {ext }}(3)$ back reacting on the proton. The re-orientation of quantum-field fluctuations of virtual pairs towards external field's direction takes place during their life-time $\delta \tau_{p} \sim 6.2 \cdot 10^{-11} \mathrm{sec}$. (corresponding to $1.86 \mathrm{~cm}$ ). The zero-point energy variation $\delta \epsilon \sim \alpha^{5} m=5.2 \cdot 10^{-6} \mathrm{eV}$, given by the Heisenberg uncertainty relationship, consistently with the rate (6) of the zero-point energy variation.

We discuss a proton passing through the vacuum. After triggered, the proton driven by the quantum-induced force moves from one spacetime point to another, virtual pairs are involved in interacting with the transverse component of proton's electric field, more and more the zero-point energy drains into the proton. As a consequence, the constant quantum-induced force, which is rather analogous to the Casimir force, is built to accelerates the proton, as if the proton gets a continuous recoil from the vacuum and rolls down along a potential with a very small slop $\sim-10^{-5} \mathrm{eV} / \mathrm{cm}$. In this spontaneous process, the proton gains the zero-point energy and the vacuum reduces its zero-point energy in such a way that the whole interacting 
system of the vacuum and proton minimizes its interacting energy. This causes the energetically favourable instability and accelerating the proton runaway.

However, any other trigger forces $\vec{F}_{t r i}^{\prime}$ acting on the proton can alter the direction of the quantum-induced force $\vec{F}_{q}$, since the later always keeps in the same direction of acceleration of the proton. Let us consider the following case: a proton driven by $\vec{F}_{q}(6)$ moves in velocity $\vec{v}$ that is in the same direction of $\vec{F}_{q}$; a trigger force $\vec{F}_{t r i}^{\prime}$ acts on such a proton in the opposite direction of $\vec{F}_{q}$ and $\left|\vec{F}_{t r i}^{\prime}\right|>\left|\vec{F}_{q}\right|$. The direction of $\vec{F}_{q}$ is altered to the direction of $\vec{F}_{t r i}^{\prime}$. After the trigger force $\vec{F}_{t r i}^{\prime}$ is off, the direction of $\vec{F}_{q}$ turns out to be opposite to the direction of proton's velocity $\vec{v}$. This causes the de-acceleration of the proton, Eq.(6) is negative for energy-lost, indicating that the kinetic energy of the proton drains back to the zeropoint energy of the vacuum. In general, this happens for $\vec{F}_{q} \cdot \vec{F}_{t r i}^{\prime}<0$ and $\left|\vec{F}_{t r i}^{\prime}\right|>\left|\vec{F}_{q}\right|$. Trigger forces $\vec{F}_{t r i}$ acting on protons are attributed to all real particles and fields, rather than virtual particles in the vacuum. With respect to a proton, these trigger forces are totally random in the spacetime. This indicates that in our Universe, some protons gain the zeropoint energy from the vacuum, whereas others instead lose their kinetic energy to the vacuum, both directions are equally probable and none of them is preferential. Our Universe is not continuously heated up by gaining the zero-point energy of the vacuum.

To discuss the energy conservation in such an spontaneously induced process of the matter and vacuum interaction, we would like to first take the Casimir effect (force) as an analogue. The Casimir force(vacuum) drives two separating plates moving closer and closer at the cost of the zero-point energy of the vacuum. On the other hand, any external force(matter) drives against the Casimir force to separate two plates moving further and further, and makes an energy-drain back to the vacuum. The induced quantum force $\vec{F}_{q}$ accelerates particles at the cost of the zero-point energy of the vacuum, and de-accelerates particles at the cost of the kinetic energy of particles. Energy-drain goes in bath directions, back and forth in between the matter and vacuum. The total energy of our Universe is conserved.

UHECRs. Based on the rate of energy-gain (6) and considering those primary protons that the energy-gain prevails in their traveling, we give a very preliminary discussion on UHECRs. With the present size of the Universe $\sim 10^{28} \mathrm{~cm}$, protons can possibly reach the energy more than $10^{21} \mathrm{eV}$, if they travel a distance $D$ of $10^{27}-10^{28} \mathrm{~cm}$ before reaching us. In such a scenario, 
primary protons, the candidates of UHECR events, could be originated from the astrophysical sources of large redshift $z$, like Quasars, or from the early Universe, and no particular arrival direction can be identified.

The GZK cutoff does not apply to such a process of protons gaining energy bit by bit on their way to us. The reason is that protons, beyond $\sim 50 \mathrm{Mpc}$ from us, have an energy much smaller than the energy threshold $10^{20} \mathrm{eV}$. This explains the absence of the GZK cutoff in UHECR events. However, when protons near us reach the energy $10^{21} \mathrm{eV}$, the GZK effect acts and average energy loss is about $\left(10^{-5}-10^{-6}\right) \mathrm{eV} / \mathrm{cm}^{2}$, which is roughly in the order of energy gain (6). This implies that ultra high energy protons would not have large possibilities to exceed the energy $10^{22} \mathrm{eV}$.

We set the origin of a spherical coordinate at the center of a primary proton's source, whose size is $R_{\circ}$, number-density $n_{\circ}$ and mean outgoing velocity $v_{\circ}$. The total flux out of the source is $4 \pi R_{\circ}^{2} n_{\circ} v_{\circ}$. The Earth is located at $R$ distance away from the source. The total flux passing through the spherical surface $4 \pi R^{2}$ is $4 \pi R^{2} n v$, where $n$ is the number-density of UHECR protons and $v$ the mean velocity. We have the conservation of total numbers of UHECR protons:

$$
4 \pi R^{2} n v=4 \pi R_{\circ}^{2} n_{\circ} v_{\circ}(1+z)^{-3},
$$

where the factor $(1+z)^{-3}$ is due to the effect of expanding Universe. Thus we obtain the flux of UHECRs measured on the Earth,

$$
\Phi=4 \pi R_{\circ}^{2} n_{\circ} v_{\circ}(1+z)^{-3} \frac{1}{4 \pi R^{2}} \sim \frac{1}{R^{2}} .
$$

Due to the distribution of intergalactic magnetic field and/or galactic wind etc, protons normally travel in a zigzag way with a mean-free path $\lambda_{p}$. The distance $D$ that protons travel is certainly larger than $R$. In one extreme case, protons travel to us in a straightforward line, $D=R$. While in

another extreme case, protons travel in a way of random walk, $D=\frac{R}{\lambda_{p}} R$. This gives rise to the spectrum of UHECR flux observed on the Earth:

$$
\Phi(E) \sim \frac{1}{R^{2}} \sim \frac{1}{E^{\gamma}} \quad 1 \leq \gamma \leq 2,
$$

where $\gamma=2$ is for proton traveling in a straightforward line $\left(\lambda_{p}=R\right)$ and $\gamma=1$ in random walk.

\section{References}

1. W. E. Lamb and R. C. Retherford, Phys. Rev. Vol. 72 (1947) 241.

2. J.W. Cronin, Nucl. Phys. (Proc. Suppl.) B28, 213 (1992). 